\title{
A survey of molecular clouds in the outer Galaxy with the highest spatial resolution
}

\author{
Mitsuhiro Matsuo ${ }^{1,2}$, Tetsuhiro Minamidani ${ }^{2,3}$, \\ Tomofumi Umemoto ${ }^{2,3}$, Atsushi Nishimura ${ }^{4}$, Hiroyuki Nakanishi ${ }^{1}$, \\ Nario Kuno ${ }^{5}$, Shinji Fujita ${ }^{2,5}$, Tomoka Tosaki ${ }^{6}$, Yuya Tsuda ${ }^{7}$, \\ Mitsuyoshi Yamagishi ${ }^{8}$, Mikito Kohno ${ }^{4}$ and the FUGIN team \\ ${ }^{1}$ Kagoshima University, email: mitsuhiro.matsuo@nao.ac.jp ${ }^{2}$ National Astronomical \\ Observatory of Japan ${ }^{3}$ SOKENDAI ${ }^{4}$ Nagoya University ${ }^{5}$ University of Tsukuba ${ }^{6}$ Joetsu \\ University of Education ${ }^{7}$ Meisei University ${ }^{8}$ Institute of Space and Astronautical Science
}

\begin{abstract}
We report a recent result of the FUGIN project, a Galactic plane CO survey using the Nobeyama 45-m Telescope and the FOREST receiver. In the third galactic quadrant, 42 square degrees are observed and 4752 molecular clouds are detected. Among them, 12 clouds are located at $R$ (distance from the Galactic center) $>16 \mathrm{kpc}$. Molecular clouds at $R<16 \mathrm{kpc}$ trace the Local, Perseus, and Outer arms.
\end{abstract}

Keywords. ISM: clouds, ISM: molecules, Galaxy: disk, Galaxy: structure, radio lines: ISM

\section{FUGIN project and the third galactic quadrant survey}

We have carried out a simultaneous survey of the $J=1-0$ transitions in ${ }^{12} \mathrm{CO},{ }^{13} \mathrm{CO}$, and $\mathrm{C}^{18} \mathrm{O}$ toward the Galactic Plane using the Nobeyama 45-m Telescope and the FOREST (FOur-beam REceiver System on the 45-m Telescope, Minamidani et al. 2016b.) as one of the legacy projects of the Nobeyama Radio Observatory. The FOREST Ultra-wide Galactic plane survey In Nobeyama (FUGIN, Umemoto et al. in prep., Minamidani et al. 2016a, Nishimura et al. 2015) project covers the areas of $l=10-50$ and $198-236$ degree for $b=-1-+1$ degree with the highest spatial resolution $\left(\sim 15^{\prime \prime}\right)$ to date, for this kind of wide-area Galactic surveys and so far, 90 square degrees have been covered.

The observed area in the third galactic quadrant is 42 square degrees. Figure 1 shows the longitude velocity diagram of the ${ }^{12} \mathrm{CO} J=1-0$ transition. We identified 4752 molecular clouds above 5 sigma noise level using CLUMPFIND algorithm. We found that 12 clouds were located at $R>16 \mathrm{kpc}$ and molecular clouds at $R<16 \mathrm{kpc}$ traced the Local, Perseus, and Outer arms. Clouds in $R>16 \mathrm{kpc}$ are obviously compact in contrast with clouds within $R<16$ kpc.

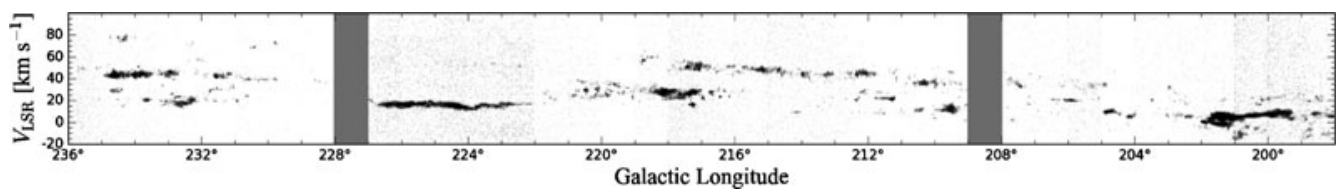

Figure 1. Longitude velocity diagram of the ${ }^{12} \mathrm{CO} J=1-0$ transition. Grey background areas have not been observed yet.

\section{References}

Minamidani, T., Umemoto, T., Nishimura, A., et al. 2016a, EAS Publications Series, 75, 193

Minamidani, T., Nishimura, A., Miyamoto, Y., et al. 2016b, Proc. SPIE, 9914, $99141 \mathrm{Z}$

Nishimura, A., Umemoto, T., Minamidani, T., et al. 2015, IAU General Assembly, 22, 2247474

Umemoto, T., Minamidani, T., Kuno, N., et al., in preparation 